\title{
A new family of azanaphthoquinones for antimicrobial evaluation
}

\author{
Nilüfer Bayrak* (1)
}

\begin{abstract}
This article presents a complete and detailed study of synthesis, structural characterization, and possible applications of a new family of azanaphthoquinones as antimicrobial agents. A series of (alkoxy)phenylamino-chloro-2-methylquinoline-5,8-dione derivatives $\left(\mathbf{3} \mathbf{a}-\mathbf{j}, \mathbf{3} \mathbf{a}^{\prime}, \mathbf{3} \mathbf{e}^{\prime}\right)$ was prepared by regioselective nucleophilic substitution of 6,7-dichloro2-methylquinoline-5,8-dione (1) with (alkoxy)arylamines $(\mathbf{2})$ in the presence of $\mathrm{CeCl}_{3} \cdot 7 \mathrm{H}_{2} \mathrm{O}$. In vitro antimicrobial study of the newly synthesized compounds was evaluated in a panel of three fungi and seven bacterial strains (three Gram-positive and four Gram-negative bacteria). As a result, the compounds ( $\mathbf{3 a}$, $\mathbf{3} \mathbf{b}$, and $\mathbf{3 h}$ ) were identified as the hits with the strong antibacterial efficiency against the human originated pathogens $\mathrm{S}$. epidermidis and $E$. faecalis with some minimal inhibitory concentration values. The antibacterial activity of the compound ( $\mathbf{3} \mathbf{h}$ ) was two times more active against $S$. epidermidis than the reference antimicrobial compound (Cefuroxime). Two compounds ( $\mathbf{3} \mathbf{a}$ and $\mathbf{3 b}$ ) exhibited excellent antibacterial activity (four times more active than Cefuroxime) against S. epidermidis. In addition to S. epidermidis, these three compounds ( $\mathbf{3} \mathbf{a}, \mathbf{3} \mathbf{b}$, and $\mathbf{3} \mathbf{h}$ ) were more active against E. faecalis than the reference antimicrobial compound (Amikacin). The antibacterial activity of the compounds ( $\mathbf{3} \mathbf{a}$ and $\mathbf{3} \mathbf{h}$ ) was three times more active against $E$. faecalis. The compound ( $\mathbf{3} \mathbf{b})$ was long dozen times more active against $E$. faecalis. For that reason, these three compounds ( $\mathbf{3} \mathbf{a}, \mathbf{3} \mathbf{b}$, and $\mathbf{3 h}$ ) were thought to be considered as the promising antibacterial agents.
\end{abstract}

Keywords: Azanaphthoquinone, Aromatic amines, Antimicrobial activity

\section{Introduction}

Looking at the outline of the existing literature on my research area to find out what are the reasons for the great importance of the compounds retaining quinoline-5,8-dione (QD) skeleton, one main section is clearly discernible. Structural and biological aspects of these derivatives make them attractive for highly successful applications, especially in pharmaceutical fields [1, 2]. These compounds are valuable and functional structures due to their possible biological activities including antibacterial, antifungal, anticancer, antimalarial, and antiviral activities [3-8]. QD skeleton has been introduced as a 'privileged template' to be a consequence of fragment-based drug discovery approaches (FDDA) that have already been penetrated into both pharmaceutical industry and academia on a large scale as an

\footnotetext{
*Correspondence: nbayrak@istanbul.edu.tr

Chemistry Department, Engineering Faculty, Istanbul University, 34320 Avcilar, Istanbul, Turkey
}

alternative methodology instead of combinatorial chemistry and high-throughput screening (HTS) techniques [9]. According to FDDA, some structural subunits that commonly found in bioactive compounds or natural substances are especially useful in design of bioeffectors $[10$, 11]. Evaluated by such a perspective of prevailing conceptual view, QD fulfills the requirement not only because of the existence of the skeleton in lavendamycin, and streptonigrin, a natural product family called "streptonigrinoids" but also due to their antimicrobial activity [12, 13]. The synthetic routes towards the important precursors of QD and its congeners have become involved in organic chemistry with special focus on a nascent class of antimicrobials.

Antimicrobials are used as a weapon to fight infectious diseases caused by germs having four main kinds, bacteria, viruses, fungi, and protozoa [14, 15]. Since these living organisms could be everywhere like air, soil, and water, the infections could easily spread by touching, eating, or even breathing [15]. According to the list of 
top 10 causes of death in the world that is declared by World Health Organization (WHO), infectious diseases such as lower respiratory tract infections, HIV/AIDS, and tuberculosis especially effecting the people most particularly in low- and middle-income countries were at the top in 2015 [16]. The principal reason for this situation is antimicrobial resistance which is described as the alteration of microorganisms at the time of their subjection to antimicrobial drugs and is usually created by the mis- and/or overuse of antimicrobials [17]. In February 2017, WHO reported a list of antibiotic-resistant priority pathogens as the greatest threat to human health containing Gram-positive and negative bacteria like Pseudomonas aeruginosa, Enterococcus faecium, Staphylococcus aureus, Klebsiella pneumonia, Escherichia coli in order to address new antibiotics that are emergently required and to encourage researchers to develop new antibiotics [17].

Our previous work on targeting synthesizing disubstituted-1,4-naphthoquinones containing a substituted arylamine and evaluating in vitro antimicrobial potential of them against Gram-positive and negative bacteria strains and fungi has led to the results that some compounds were identified as the hits with the strong antibacterial efficiency against the human originated pathogens S. epidermidis exhibiting excellent antibacterial activity [18]. When the toxicity of some compounds was compared with the reference clinically proven antimicrobial drug Cefuroxime, the antibacterial activities of some compounds were 2-4 times more active than Cefuroxime [18]. By the evaluation of the studies related to the structure-activity relationships on QD and its derivatives, the clear evidence was observed that the antimicrobial activity has been associated with the structural diversity at the benzyl chain or/and at the quinoline ring of the QD core. Based on the facts infections are among the top ten causes of morbidity and mortality worldwide and due to the high degree of resistance of germs by the way putting a bridle on the therapy of the infections, I have paid particular attention to synthesize new organic compounds comprising QD skeleton as antimicrobial drug candidates with the hope of circumventing the vital threats and creating new antibiotics that are urgently needed.

\section{Results and discussion Chemistry}

Keeping in mind the previous researches on the regioselective nucleophilic substitution of dichloro quinoline-5,8-dione with amines, new 6-arylamino7-chloro-5,8-quinazolinedione analogues have been synthesized using $\mathrm{CeCl}_{3} \cdot 7 \mathrm{H}_{2} \mathrm{O}$ and ethanol as reported before [19]. What we do know from existing literature is that the nucleophilic substitution of dichloro-2-methylquinoline-5,8-dione compound (1) containing two unsymmetrically substituted chlorine atoms which are capable of replacing amines can occur at the C-6 and/ or $\mathrm{C}-7$ position depending on the reaction parameters. By adding a Lewis acid like $\mathrm{CeCl}_{3} \cdot 7 \mathrm{H}_{2} \mathrm{O}$, the regioselectivity of 6-position is significantly increased owing to decreased electron density at C-6 via the chelation of $\mathrm{Ce}(\mathrm{III})$ between heterocyclic nitrogen atom and oxygen atom at C-8 position. As a result, the chlorine atom in position 6 is more inclinable to be substituted than that in position 7 [19]. Besides, polar solvents such as ethanol are also known to enhance the formation of 6-isomer products.

Starting material 6,7-dichloro-2-methylquinoline5,8-dione (1) was synthesized according to a procedure described in the literature [20]. The quinoline $\mathbf{1}$ was reacted analogously with commercial amines such as 4-methoxybenzenamine, 3-methoxybenzenamine, 2-methoxybenzenamine, 4-ethoxybenzenamine, 3-ethoxybenzenamine, 2-ethoxybenzenamine, 2,4-dimethoxybenzenamine, 2,5-dimethoxybenzenamine, 3,4-dimethoxybenzenamine, and 3,5-dimethoxybenzenamine to give 6-alkoxyphenylamino-7-chloroquinoline diones $(\mathbf{3 a}-\mathbf{j})$ as the main products except two compounds named $\mathbf{3} \mathbf{a}^{\prime}$ and $3 \mathbf{e}^{\prime}$ that are 7-alkoxyphenylamino-6-chloroquinoline diones. The reaction mixtures of products $\left(\mathbf{3} \mathbf{a}-\mathbf{j}, 3 \mathbf{a}^{\prime}\right.$, and $\left.3 \mathbf{e}^{\prime}\right)$ were then subjected for purification using column chromatography. The chemical structures of the derivatives were confirmed by ${ }^{1} \mathrm{H}$ and ${ }^{13} \mathrm{C}$ NMR, IR, and MS methods. The synthesis of compounds $\left(\mathbf{3} \mathbf{a}-\mathbf{j}, \mathbf{3} \mathbf{a}^{\prime}\right.$, and $\left.\mathbf{3} \mathbf{e}^{\prime}\right)$ were depicted in Scheme 1.

As a matter of fact, it is observed the characteristic signals of the IR spectra of $\mathbf{3 a}-\mathbf{j}, \mathbf{3} \mathbf{a}^{\prime}, \mathbf{3} \mathbf{e}^{\prime}$ showed characteristic absorptions at around $3200 \mathrm{~cm}^{-1}(\mathrm{NH}) ; 1650 \mathrm{~cm}^{-1}$ $(\mathrm{C}=\mathrm{O}), 2900 \mathrm{~cm}^{-1}\left(\mathrm{CH}_{\text {aliphatic }}\right)$. The ${ }^{1} \mathrm{H}$ NMR spectra of all synthesized compounds exhibited the $\mathrm{NH}$ signals as a singlet at around $7.60 \mathrm{ppm}$. The aromatic proton resonances appeared in a range of 6-8 ppm. The signals related to other functional groups observed in the expected regions.

\section{Antimicrobial activity}

A previous study was aimed to investigate the interaction of 6-chloroquinoline-5,8-dione hydrochloride and 6,7-dichloroquinoline-5,8-quinone with sulfa drugs such as sulfadiazine, sulfadimidine, and sulfathiazole. In that study, the effect of products characterized as 7 -substituted sulfonamide $10 \mathrm{H}$-pyrido[3,2-b]carbazole5,11-dione and 6-substituted sulfonamide derivatives of 7-chloroquinoline-5,8-quinone on some Gram positive and Gram negative bacterial species were tested [21]. To better understand how the antifungal activity might be 


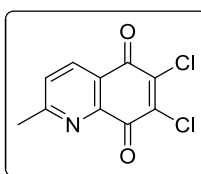

1

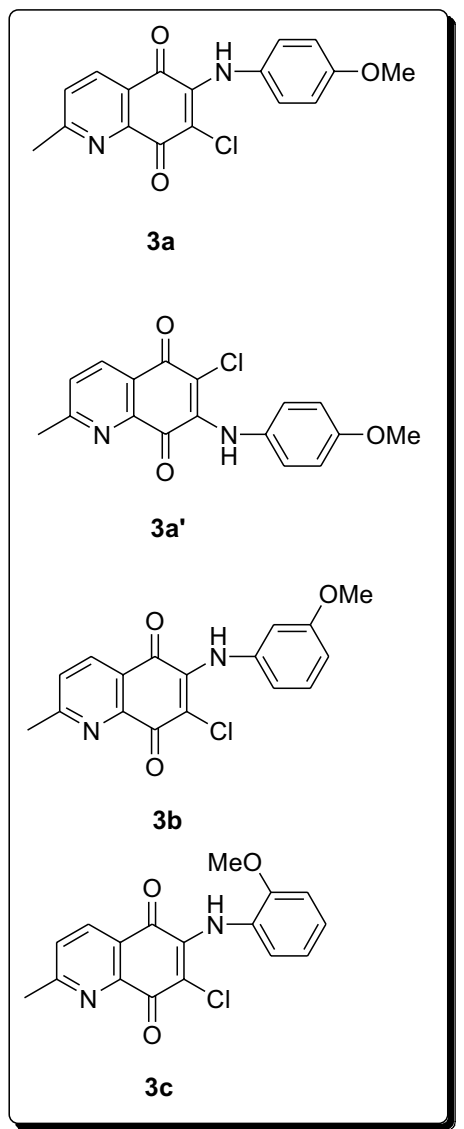

EtOH/Reflux

$\mathrm{CeCl}_{3} \cdot 7 \mathrm{H}_{2} \mathrm{O}$

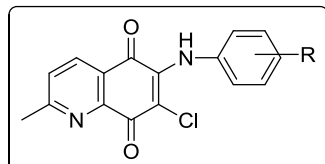

$3 a-j$<smiles>CCOc1ccc(NC2=C(Cl)C(=O)c3nc(C)ccc3C2=O)cc1</smiles><smiles>CCOc1cccc(NC2=C(Cl)C(=O)c3nc(C)ccc3C2=O)c1</smiles><smiles>CCOc1cccc(NC2=C(Cl)C(=O)c3ccc(C)nc3C2=O)c1</smiles>

$3 e^{\prime}$

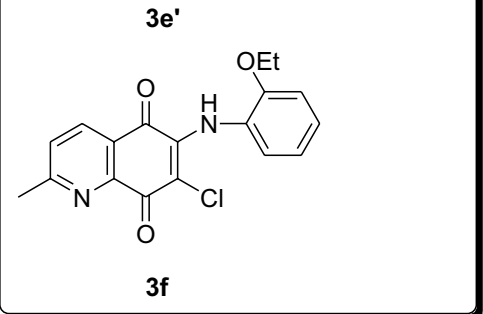

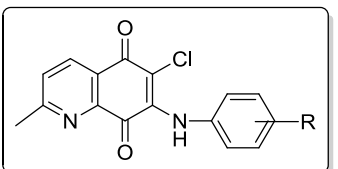

$3 a^{\prime}$ and 3e'<smiles>COc1ccc(NC2=C(Cl)C(=O)c3nc(C)ccc3C2=O)c(OC)c1</smiles>

3h<smiles>COc1ccc(NC2=C(Cl)C(=O)c3nc(C)ccc3C2=O)cc1OC</smiles>

$3 \mathbf{i}$

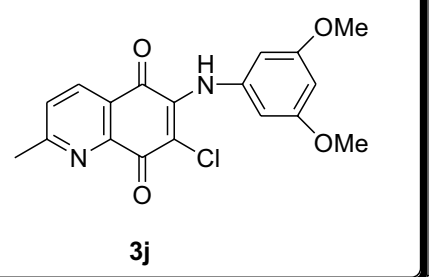

Scheme 1 The library of new family of azanaphthoquinones

influenced by both the number and the position of halogen or other substituents on the aniline ring, it would be helpful to look over the reports related to the synthesis of 6-[N-(substitutedphenyl)amino]-7-chloro-5,8-quinolinediones to use for their antifungal susceptibility testings, in vitro, against pathogenic Candida species and bacteria. Some derivatives of these compounds showed more potent antifungal activities than ketoconazole, fluconazole and griseofulvin used for antifungal references in these studies. Most of derivatives were found to be more active than ampicillin against gram-positive bacteria. Moreover, 6-[(N-2,3-dichlorophenyl)amino]7-chloro-5,8-quinolinedione was tested for their in vivo antifungal activities in the treatment of systemic infection with Candida albicans in normal mice compared with ketoconazole and it was declared as a potent antifungal agent $[4,22]$.

To explore the antibacterial potential of the synthesized QDs, total twelve compounds were evaluated in vitro for their antibacterial activity against gram-positive and gramnegative bacteria and for their antifungal activity against fungi using the microbroth dilution technique according to the Clinical Laboratory Standards Institute (CLSI) recommendations $[23,24]$. The antimicrobial assay results of all the newly synthesized QDs $\left(\mathbf{3} \mathbf{a}-\mathbf{j}, \mathbf{3} \mathbf{a}^{\prime}\right.$, and $\left.\mathbf{3} \mathbf{e}^{\prime}\right)$ are given in Table 1 . Concerning the antibacterial activity, the results showed that some compounds displayed versatile effects on the growth of the tested gram-positive and gram-negative bacterial strains. The test-cultures $P$. aeruginosa and $P$. mirabilis appeared not to be susceptible to synthesized 


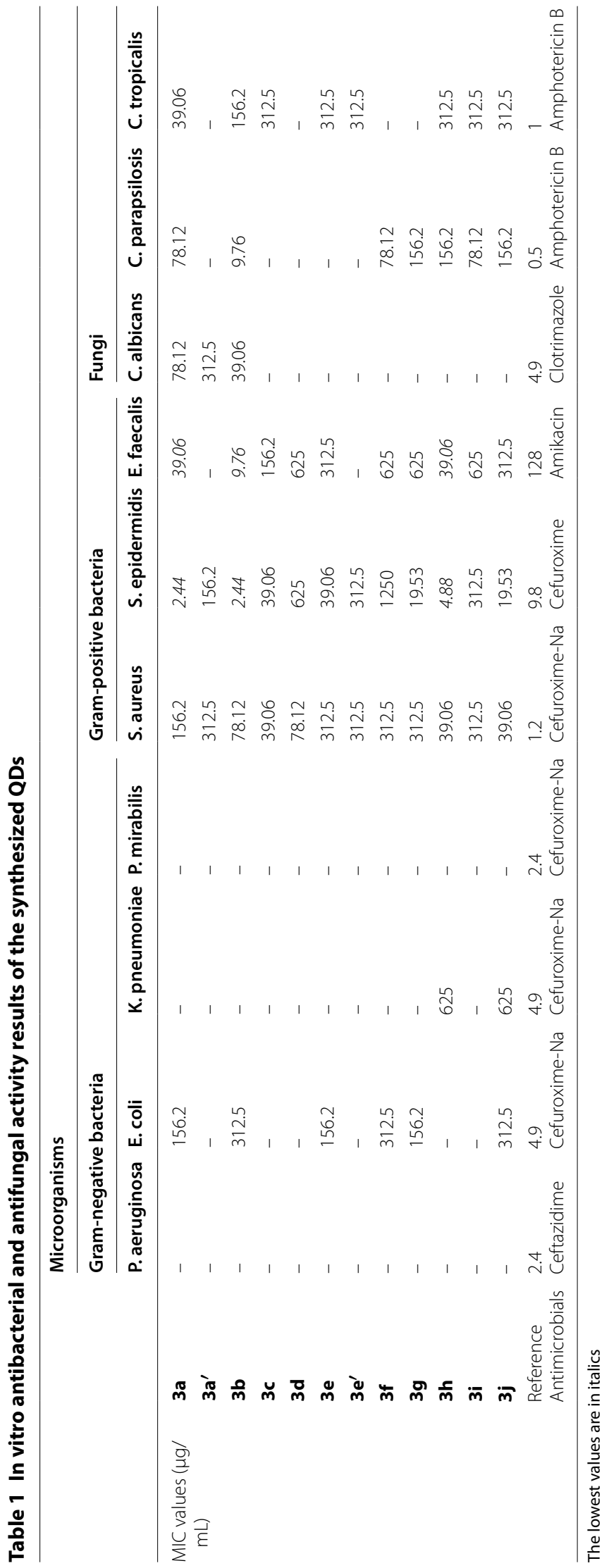


compounds. Findings shown in Table 1 revealed that compounds have exhibited moderate activity against both Gram-positive bacteria. All of the synthesized compounds possessed activity against $S$. aureus and $S$. epidermidis with MIC values of between 2.44 and $1250 \mu \mathrm{g} / \mathrm{mL}$. Additionally, all of the compounds, except $\mathbf{3} \mathbf{a}^{\prime}$, and $\mathbf{3} \mathbf{e}^{\prime}$, possessed activity against $E$. faecalis with MIC values of between 9.76 and $625 \mu \mathrm{g} / \mathrm{mL}$. Compounds 3a, $3 \mathbf{b}$, and $\mathbf{3 h}$ showed excellent activity against $S$. epidermidis and $E$. faecalis. These results are important considering the intrinsic resistance to Cefuroxime and Amikacin of S. epidermidis and E. faecalis, respectively. These three compounds showed fairly wide antifungal spectra. The activities of the compounds 3a, $\mathbf{3 b}$, and $\mathbf{3 h}$ were superior as compared to that of Cefuroxime. The compounds $\mathbf{3 a}, \mathbf{3 b}$, and $\mathbf{3 h}$, completely inhibited the growth of $S$. epidermidis, was tested at the MIC level of $2.44,2.44$, and $4.88 \mu \mathrm{g} / \mathrm{mL}$, respectively. By contrast, 7 -isomer products of $\mathbf{3 a}$ and $\mathbf{3 e}$, namely $\mathbf{3 \mathbf { a } ^ { \prime }}$ and $\mathbf{3} \mathbf{e}^{\prime}$, has no significant activity against $S$. epidermidis and $E$. faecalis. In addition to these, the activities of compounds $\mathbf{3 g}$ and $\mathbf{3 j}$ showed moderate activity against S. epidermidis.

To evaluate the importance of the position and alkyl chain of alkoxy group (-OR) on the phenyl amine ring with respect to the biological efficiency, the activities of all the synthesized compounds were compared (Scheme 1). It was found that replacing the methoxy group $\left(-\mathrm{OCH}_{3}\right)$ position in the synthesized compounds $(3 \mathbf{a}-\mathbf{c})$ from the meta position to the para position or the meta position to the ortho position led to activity loss. Additionally, replacing the methoxy group $\left(-\mathrm{OCH}_{3}\right)$ by an ethoxy group $\left(-\mathrm{OCH}_{2} \mathrm{CH}_{3}\right)$ in all positions did not show any positive progress on activity. Presence of the two methoxy groups in different positions such as 2,4-, 2,5-, 3,4-, and 3,5-(3g-j) led to a decrease in activity when compared with compounds $(\mathbf{3 a}-\mathbf{c})$ containing only one methoxy group. On the other hand, presence of the two methoxy groups $(\mathbf{3 g}-\mathbf{j})$ has still much more active than the compounds $(\mathbf{3 d}-\mathbf{f})$ containing ethoxy group. According to this data, we can conclude that, in general, the QDs containing one methoxy group showed better antibacterial activity than their ethoxy derivatives.

\section{Materials and methods \\ Chemistry}

All reagents and solvents were commercially purchased from different companies and used as supplied. Chemical reactions were monitored by using aluminium-based plates (silica gel 60 F254) from Merck. Plates were visualized under UV light (254 nm). Column chromatographic separations were carried out using silica gel 60 (Merck, 63-200 $\mu \mathrm{m}$ particle size, 60-230 mesh). Melting points (mp) were determined with a Buchi B-540 melting point without being corrected. ${ }^{1} \mathrm{H}$ NMR and ${ }^{13} \mathrm{C}$ NMR spectra were recorded with either VarianUNITY INOVA spectrometers with $500 \mathrm{MHz}$ frequency for ${ }^{1} \mathrm{H}$ and $125 \mathrm{MHz}$ frequency for ${ }^{13} \mathrm{C}$ NMR or BRUKER spectrometers with $400 \mathrm{MHz}$ frequency for ${ }^{1} \mathrm{H}$ and $100 \mathrm{MHz}$ frequency for ${ }^{13} \mathrm{C}$ NMR in ppm. ${ }^{1} \mathrm{H}$ NMR spectra and ${ }^{13} \mathrm{C}$ NMR spectra in $\mathrm{CDCl}_{3}$ refer to the solvent signal center at 7.19 and $76.0 \mathrm{ppm}$, respectively. Chemical shifts are expressed in ppm downfield relative to tetramethylsilane. Data for ${ }^{1} \mathrm{H}$ NMR spectra are reported as follows: $\mathrm{s}$ (singlet), br s (broad singlet), d (doublet), t (triplet), q (quartet), dd (doublet of doublets), and $\mathrm{m}$ (multiplet) and the coupling constants J are given in Hz. IR spectra were recorded as ATR on either Thermo Scientific Nicolet 6700 spectrometer or Alpha T FTIR spectrometer. Mass spectra were obtained on either a Thermo Finnigan LCQ Advantage MAX MS/MS spectrometer equipped with ESI (electrospray ionization) sources. (Alkoxyphenylamino)-chloro2-methylquinoline-5,8-dione derivatives $\left(\mathbf{3} \mathbf{a}-\mathbf{j}, \mathbf{3 \mathbf { a } ^ { \prime }}\right.$, and $\left.3 \mathbf{e}^{\prime}\right)$ were prepared from the reactions of 6,7 -dichloro2-methylquinoline-5,8-dione (1) with alkoxy substituted aryl amines $(\mathbf{2} \mathbf{a}-\mathbf{j})$ according to the procedure previously reported [19].

\section{General procedure for synthesis of the (alkoxyphenylamino)-chloro-2-methylquinoline-5,8-dione derivatives $\left(3 a-j, 3 a^{\prime}, 3 e^{\prime}\right)$}

Into a $100 \mathrm{~mL}$ round bottom flask were added $250 \mathrm{mg}$ (1.00 mmol) of 6,7-dichloro-2-methylquinoline-5,8-dione (1), ethanol $(30 \mathrm{~mL}), \mathrm{CeCl}_{3} \cdot 7 \mathrm{H}_{2} \mathrm{O}$, and alkoxy substituted aryl amines (2), respectively. The reaction mixture was stirred at room temperature until thin layer chromatography (TLC) showed the absence of the starting materials. The resulting solution was extracted with $100 \mathrm{~mL}$ chloroform then washed with water $(3 \times 100 \mathrm{~mL})$ and dried over calcium chloride. The solvent was removed in vacuo. The residue was subjected to column chromatography on silica gel using suitable solvents to give the products (3a-j, $\mathbf{3} \mathbf{a}^{\prime}$, and $\left.\mathbf{3} \mathbf{e}^{\prime}\right)$ [19].

6-(4-Methoxyphenylamino)-7-chloro-2-methylquinoline-5,8-dione (3a) It was synthesized from 6,7-dichloro2-methylquinoline-5,8-dione (1) and 4-methoxybenzenamine (2a) as purple powder by using the general procedure. Yield: $0.1 \mathrm{~g}, 30 \%$, mp: $189-190^{\circ} \mathrm{C}$. FTIR (ATR) $v\left(\mathrm{~cm}^{-1}\right)$ : $3234(-\mathrm{NH}), 2967,2938,2855\left(\mathrm{CH}_{\text {aliphatic }}\right), 1678$, $1649(\mathrm{C}=\mathrm{O}), 1580(\mathrm{C}=\mathrm{C}) .{ }^{1} \mathrm{H}$ NMR (500 MHz, $\left.C D C l_{3}\right)$ $\delta$ (ppm): 8.30 (d, J: $\left.7.8 \mathrm{~Hz}, 1 \mathrm{H},-\mathrm{CH}_{\text {arom }}\right), 7.63($ br s, $1 \mathrm{H}$, $-\mathrm{NH}), 7.48\left(\mathrm{~d}, \mathrm{~J}: 7.8 \mathrm{~Hz}, 1 \mathrm{H},-\mathrm{CH}_{\text {arom }}\right), 7.10-7.04(\mathrm{~m}$, $\left.2 \mathrm{H},-\mathrm{CH}_{\text {arom }}\right), 6.94-6.86\left(\mathrm{~m}, 2 \mathrm{H},-\mathrm{CH}_{\text {arom }}\right), 3.85(\mathrm{~s}, 3 \mathrm{H}$, $\left.-\mathrm{OCH}_{3}\right), 2.80\left(\mathrm{~s}, 3 \mathrm{H},-\mathrm{CH}_{3}\right) \cdot{ }^{13} \mathrm{C} \mathrm{NMR}\left(125 \mathrm{MHz}, \mathrm{CDCl}_{3}\right)$ $\delta(\mathrm{ppm}): 180.1,176.1(\mathrm{C}=\mathrm{O}), 166.0,158.0,148.1,141.2$, $134.9,130.9,126.7,126.3,124.6,114.4,113.7\left(C_{q}\right.$ and 
$\left.\mathrm{C}_{\text {arom }}\right), 55.5\left(-\mathrm{OCH}_{3}\right), 25.4\left(-\mathrm{CH}_{3}\right)$. MS (ESI+) $m / z(\%)$ : $328\left(100,[\mathrm{M}]^{+}\right)$. Anal. Calcd. for $\mathrm{C}_{17} \mathrm{H}_{13} \mathrm{ClN}_{2} \mathrm{O}_{3}(328.75)$.

Additionally, $\mathbf{3 \mathbf { a } ^ { \prime }}$ was also obtained from 6,7-dichloro2-methylquinoline-5,8-dione (1) and 4-methoxybenzenamine (2a) as purple powder by using the general procedure.

7-(4-Methoxyphenylamino)-6-chloro-2-methylquinoline-5,8-dione (3a') Yield: $0.034 \mathrm{~g}, 10 \%$. mp: $215-216{ }^{\circ} \mathrm{C}$. FTIR (ATR) $v\left(\mathrm{~cm}^{-1}\right): 3226(-\mathrm{NH}), 3002,2969,2851$ $\left(\mathrm{CH}_{\text {aliphatic }}\right), 1678,1652(\mathrm{C}=\mathrm{O}), 1609,1580(\mathrm{C}=\mathrm{C}) .{ }^{1} \mathrm{H}$ $\operatorname{NMR}\left(400 \mathrm{MHz}, C D C l_{3}\right) \delta(\mathrm{ppm}): 8.31(\mathrm{~d}, J: 8.0 \mathrm{~Hz}, 1 \mathrm{H}$, $\left.-\mathrm{CH}_{\text {arom }}\right), 7.67($ br s, $1 \mathrm{H},-\mathrm{NH}), 7.48(\mathrm{~d}, \mathrm{~J}: 8.0 \mathrm{~Hz}, 1 \mathrm{H},-$ $\left.\mathrm{CH}_{\text {arom }}\right), 7.00\left(\mathrm{~d}, \mathrm{~J}: 8.8 \mathrm{~Hz}, 2 \mathrm{H},-\mathrm{CH}_{\text {arom }}\right), 6.81(\mathrm{~d}, \mathrm{~J}: 8.9 \mathrm{~Hz}$, $\left.2 \mathrm{H},-\mathrm{CH}_{\text {arom }}\right), 3.50\left(\mathrm{~s}, 3 \mathrm{H},-\mathrm{OCH}_{3}\right), 2.70\left(\mathrm{~s}, 3 \mathrm{H},-\mathrm{CH}_{3}\right)$.

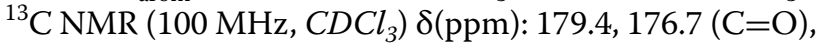
$164.0,158.0,146.0,142.0,135.1,130.0,128.6,128.0$, 126.5, 113.6, $112.5\left(\mathrm{C}_{\mathrm{q}}\right.$ and $\left.\mathrm{C}_{\text {arom }}\right), 55.5\left(-\mathrm{OCH}_{3}\right), 25.1(-$ $\left.\mathrm{CH}_{3}\right)$. MS (ESI+) $m / z(\%): 329\left(100,[\mathrm{M}+\mathrm{H}]^{+}\right), 331(32$, $\left.[\mathrm{M}+3 \mathrm{H}]^{+}\right)$. Anal. Calcd. for $\mathrm{C}_{17} \mathrm{H}_{13} \mathrm{ClN}_{2} \mathrm{O}_{3}(328.75)$.

6-(3-Methoxyphenylamino)-7-chloro-2-methylquinoline-5,8-dione (3b) It was synthesized from 6,7-dichloro2-methylquinoline-5,8-dione (1) and 3-methoxybenzenamine (2b) as purple oil by using the general procedure. Yield: $0.050 \mathrm{~g}, 15 \%$. FTIR (ATR) $v\left(\mathrm{~cm}^{-1}\right): 3426(-\mathrm{NH})$, 2971, 2934, 2985, $2859\left(\mathrm{CH}_{\text {aliphatic }}\right), 1678,1652,(\mathrm{C}=\mathrm{O})$, $1576(\mathrm{C}=\mathrm{C}) .{ }^{1} \mathrm{H}$ NMR $\left(400 \mathrm{MHz}, \mathrm{CDCl}_{3}\right) \delta(\mathrm{ppm}): 8.34$ (d, J: $\left.8.0 \mathrm{~Hz}, 1 \mathrm{H},-\mathrm{CH}_{\text {arom }}\right), 7.67($ br s, $1 \mathrm{H},-\mathrm{NH}), 7.52(\mathrm{~d}$, : $:$ $\left.8.0 \mathrm{~Hz}, 1 \mathrm{H},-\mathrm{CH}_{\text {arom }}\right), 7.28\left(\mathrm{t}, \mathrm{J}: 8.1 \mathrm{~Hz}, 1 \mathrm{H},-\mathrm{CH}_{\text {arom }}\right), 6.82$ (dd, J: 8.2, J: $2.3 \mathrm{~Hz}, 1 \mathrm{H},-\mathrm{CH}_{\text {arom }}$ ), 6.72 (dd, J: 9,8, J: $2.0 \mathrm{~Hz}$, $\left.1 \mathrm{H},-\mathrm{CH}_{\text {arom }}\right), 6.65\left(\mathrm{t}, \mathrm{J}: 2.2 \mathrm{~Hz}, 1 \mathrm{H},-\mathrm{CH}_{\text {arom }}\right), 3.84(\mathrm{~s}, 3 \mathrm{H}$, $\left.-\mathrm{OCH}_{3}\right), 2.82\left(\mathrm{~s}, 3 \mathrm{H},-\mathrm{CH}_{3}\right) \cdot{ }^{13} \mathrm{C} \mathrm{NMR}\left(100 \mathrm{MHz}, \mathrm{CDCl}_{3}\right)$ $\delta(\mathrm{ppm}): 180.1,176.2(\mathrm{C}=\mathrm{O}), 166.1,159.7,148.0,140.9$, 138.3, 135.0, 129.2, 126.9, 124.7, 116.8, 116.3, 111.7, 110.2 $\left(\mathrm{C}_{\mathrm{q}}\right.$ and $\left.\mathrm{C}_{\text {arom }}\right), 55.5\left(-\mathrm{OCH}_{3}\right), 25.5\left(-\mathrm{CH}_{3}\right) . \mathrm{MS}(\mathrm{ESI}+)$ $m / z(\%): 329\left(100,[\mathrm{M}+\mathrm{H}]^{+}\right), 331\left(28,[\mathrm{M}+3 \mathrm{H}]^{+}\right)$. Anal. Calcd. for $\mathrm{C}_{17} \mathrm{H}_{13} \mathrm{ClN}_{2} \mathrm{O}_{3}$ (328.75).

6-(2-Methoxyphenylamino)-7-chloro-2-methylquinoline-5,8-dione (3c) It was synthesized from 6,7-dichloro2-methylquinoline-5,8-dione (1) and 2-methoxybenzenamine (2c) as dark red powder by using the general procedure. Yield: $0.179 \mathrm{~g}, 53 \%$, mp: $210-211{ }^{\circ} \mathrm{C}$. FTIR (ATR) $v\left(\mathrm{~cm}^{-1}\right): 3224(-\mathrm{NH}), 2998,2994,2845\left(\mathrm{CH}_{\text {aliphatic }}\right)$, 1676, $1652(\mathrm{C}=\mathrm{O}), 1607,1576(\mathrm{C}=\mathrm{C}) .{ }^{1} \mathrm{H}$ NMR $(400 \mathrm{MHz}$, $\left.\mathrm{CDCl}_{3}\right) \delta(\mathrm{ppm}): 8.33$ (d, J: $\left.7.9 \mathrm{~Hz}, 1 \mathrm{H},-\mathrm{CH}_{\text {arom }}\right), 7.63(\mathrm{br}$ s, $1 \mathrm{H},-\mathrm{NH}), 7.51\left(\mathrm{~d}, \mathrm{~J}: 7.9 \mathrm{~Hz}, 1 \mathrm{H},-\mathrm{CH}_{\mathrm{arom}}\right), 7.27-7.22$ $\left(\mathrm{m}, 1 \mathrm{H},-\mathrm{CH}_{\text {arom }}\right), 7.07-6.92\left(\mathrm{~m}, 3 \mathrm{H},-\mathrm{CH}_{\text {arom }}\right), 3.88(\mathrm{~s}$, $\left.3 \mathrm{H},-\mathrm{OCH}_{3}\right), 2.82\left(\mathrm{~s}, 3 \mathrm{H},-\mathrm{CH}_{3}\right) .{ }^{13} \mathrm{C}$ NMR $(100 \mathrm{MHz}$, $\left.\mathrm{CDCl}_{3}\right) \delta(\mathrm{ppm}):$ 180.0, $176.1(\mathrm{C}=\mathrm{O}), 166.0,152.6,148.1$, 141.2, 135.0, 126.8, 126.1, 125.2, 124.8, 119.9, 115.4, 110.7 $\left(\mathrm{C}_{\mathrm{q}}\right.$ and $\left.\mathrm{C}_{\text {arom }}\right), 55.7\left(-\mathrm{OCH}_{3}\right), 25.6\left(-\mathrm{CH}_{3}\right) . \mathrm{MS}(\mathrm{ESI}+)$ $m / z(\%): 329\left(100,[\mathrm{M}+\mathrm{H}]^{+}\right), 331\left(22,[\mathrm{M}+3 \mathrm{H}]^{+}\right)$. Anal. Calcd. for $\mathrm{C}_{17} \mathrm{H}_{13} \mathrm{ClN}_{2} \mathrm{O}_{3}$ (328.75).

6-(4-Ethoxyphenylamino)-7-chloro-2-methylquinoline-5,8-dione (3d) It was synthesized from 6,7-dichloro-2-methylquinoline-5,8-dione (1) and 4-ethoxybenzenamine (2d) as purple powder by using the general procedure. Yield: $0.165 \mathrm{~g}, 47 \%$, mp: $193-194{ }^{\circ} \mathrm{C}$. FTIR (ATR) $v\left(\mathrm{~cm}^{-1}\right): 3224(-\mathrm{NH}), 2998,2969,2855\left(\mathrm{CH}_{\text {aliphatic }}\right)$, 1676, $1652(\mathrm{C}=\mathrm{O}), 1607,1578(\mathrm{C}=\mathrm{C}) .{ }^{1} \mathrm{H}$ NMR $(400 \mathrm{MHz}$, $\left.\mathrm{CDCl}_{3}\right) \delta(\mathrm{ppm}): 8.21\left(\mathrm{~d}, \mathrm{~J}: 8.00 \mathrm{~Hz}, 1 \mathrm{H},-\mathrm{CH}_{\text {arom }}\right), 7.52(\mathrm{br}$ s, $1 \mathrm{H},-\mathrm{NH}), 7.40\left(\mathrm{~d}, \mathrm{~J}: 8.0 \mathrm{~Hz}, 1 \mathrm{H},-\mathrm{CH}_{\text {arom }}\right), 7.16-7.07$ (m, $\left.1 \mathrm{H},-\mathrm{CH}_{\text {arom }}\right), 7.00-6.92\left(\mathrm{~m}, 1 \mathrm{H},-\mathrm{CH}_{\text {arom }}\right), 6.88-6.78$ $\left(\mathrm{m}, 2 \mathrm{H},-\mathrm{CH}_{\text {arom }}\right), 4.00$ (q, J: 13.9, $\left.6.9 \mathrm{~Hz}, 2 \mathrm{H},-\mathrm{OCH}_{2}\right)$, $2.71\left(\mathrm{~s}, 3 \mathrm{H},-\mathrm{CH}_{3}\right), 1.27\left(\mathrm{t}, \mathrm{J}: 7.0 \mathrm{~Hz}, 3 \mathrm{H},-\mathrm{CH}_{3}\right) .{ }^{13} \mathrm{C} \mathrm{NMR}$ $\left(100 \mathrm{MHz}, \mathrm{CDCl}_{3}\right) \delta(\mathrm{ppm}): 179.9,176.0(\mathrm{C}=\mathrm{O}), 165.9$, 152.0, 148.1, 141.4, 134.9, 126.7, 126.3, 125.1, 124.8, 119.8, $115.5,111.6\left(\mathrm{C}_{\mathrm{q}}\right.$ and $\left.\mathrm{C}_{\text {arom }}\right), 64.2\left(-\mathrm{OCH}_{2}\right), 25.5,14.8(-$ $\left.\mathrm{CH}_{3}\right)$. MS (ESI-) $m / z(\%): 341\left(100,[\mathrm{M}-\mathrm{H}]^{-}\right), 343$ (30, $\left.[\mathrm{M}+\mathrm{H}]^{-}\right)$. Anal. Calcd. for $\mathrm{C}_{18} \mathrm{H}_{15} \mathrm{ClN}_{2} \mathrm{O}_{3}(342.78)$.

6-(3-Ethoxyphenylamino)-7-chloro-2-methylquinoline-5,8-dione (3e) It was synthesized from 6,7-dichloro2-methylquinoline-5,8-dione (1) and 3-ethoxybenzenamine (2e) as dark red powder by using the general procedure. Yield: $0.157 \mathrm{~g}, 44 \%$, mp: $187-188{ }^{\circ} \mathrm{C}$. FTIR (ATR) $v\left(\mathrm{~cm}^{-1}\right): 3330(-\mathrm{NH}), 2969,2928,2861\left(\mathrm{CH}_{\text {aliphatic }}\right)$, $1678,1666(\mathrm{C}=\mathrm{O}), 1603,1580(\mathrm{C}=\mathrm{C}) .{ }^{1} \mathrm{H}$ NMR $(500 \mathrm{MHz}$, $\left.\mathrm{CDCl}_{3}\right) \delta(\mathrm{ppm}): 8.22\left(\mathrm{~d}, \mathrm{~J}: 7.8 \mathrm{~Hz}, 1 \mathrm{H},-\mathrm{CH}_{\text {arom }}\right), 7.55$ (br s, $1 \mathrm{H},-\mathrm{NH}), 7.40\left(\mathrm{~d}, J: 7.8 \mathrm{~Hz}, 1 \mathrm{H},-\mathrm{CH}_{\text {arom }}\right), 7.16(\mathrm{t}$, J: $8.3 \mathrm{~Hz}, 1 \mathrm{H},-\mathrm{CH}_{\text {arom }}$ ), 6.69 (dd, J: 8.3, J: $2.4 \mathrm{~Hz}, 1 \mathrm{H}$, $\left.\mathrm{CH}_{\text {arom }}\right), 6.59$ (dd, J: 7.8, J: $\left.1.9 \mathrm{~Hz}, 1 \mathrm{H},-\mathrm{CH}_{\text {arom }}\right), 6.54-6.53$ $\left(\mathrm{m}, 1 \mathrm{H},-\mathrm{CH}_{\text {arom }}\right), 3.98-3.93\left(\mathrm{~m}, 2 \mathrm{H},-\mathrm{OCH}_{2}\right), 2.71(\mathrm{~s}, 3 \mathrm{H}$, $\left.-\mathrm{CH}_{3}\right), 1.33\left(\mathrm{t}, \mathrm{J}: 6.8 \mathrm{~Hz}, 3 \mathrm{H},-\mathrm{CH}_{3}\right) .{ }^{13} \mathrm{C} \mathrm{NMR}(125 \mathrm{MHz}$, $\left.\mathrm{CDCl}_{3}\right) \delta(\mathrm{ppm}):$ 179.1, $175.1(\mathrm{C}=\mathrm{O}), 165.1,158.1,147.0$, 139.9, 137.3, 133.9, 128.1, 125.8, 123.7, 115.7, 115.0, 111.3, $109.8\left(\mathrm{C}_{\mathrm{q}}\right.$ and $\left.\mathrm{C}_{\text {arom }}\right), 62.7\left(-\mathrm{OCH}_{2}\right), 24.4,13.8\left(-\mathrm{CH}_{3}\right) . \mathrm{MS}$ $(\mathrm{ESI}-) \mathrm{m} / z(\%): 341\left(100,[\mathrm{M}-\mathrm{H}]^{-}\right), 343\left(32,[\mathrm{M}+\mathrm{H}]^{-}\right)$. Anal. Calcd. for $\mathrm{C}_{18} \mathrm{H}_{15} \mathrm{ClN}_{2} \mathrm{O}_{3}$ (342.78).

Additionally, $3 \mathbf{e}^{\prime}$ was also obtained from 6,7-dichloro2-methylquinoline-5,8-dione (1) and 3-ethoxybenzenamine (2e) as red oil by using the general procedure.

7-(3-Ethoxyphenylamino)-6-chloro-2-methylquinoline-5,8-dione $\left(3 \boldsymbol{e}^{\prime}\right)$ Yield: $0.010 \mathrm{~g}, 3 \%$. FTIR (ATR) $v\left(\mathrm{~cm}^{-1}\right): 3442(-\mathrm{NH}), 2965,2926,2857,\left(\mathrm{CH}_{\text {aliphatic }}\right), 1637$ $(\mathrm{C}=\mathrm{O}), 1509,1466(\mathrm{C}=\mathrm{C}) .{ }^{1} \mathrm{H}$ NMR $\left(500 \mathrm{MHz}, C D C l_{3}\right) \delta$ (ppm): 8.31 (d, J: $8.3 \mathrm{~Hz}, 1 \mathrm{H},-\mathrm{CH}_{\text {arom }}$ ), $7.69(\mathrm{br} \mathrm{s}, 1 \mathrm{H}$, $\mathrm{NH}), 7.47\left(\mathrm{~d}, J: 7.8 \mathrm{~Hz}, 1 \mathrm{H},-\mathrm{CH}_{\text {arom }}\right), 7.17(\mathrm{t}, \mathrm{J}: 7.8 \mathrm{~Hz}, 1 \mathrm{H}$, $\left.-\mathrm{CH}_{\text {arom }}\right), 6.70\left(\mathrm{dd}, \mathrm{J:} 8.3,2.4 \mathrm{~Hz}, 1 \mathrm{H},-\mathrm{CH}_{\text {arom }}\right), 6.62(\mathrm{dd}$, J: $\left.7.8,1.9 \mathrm{~Hz}, 1 \mathrm{H},-\mathrm{CH}_{\text {arom }}\right), 6.56-6.55\left(\mathrm{~m}, 1 \mathrm{H},-\mathrm{CH}_{\text {arom }}\right)$, $3.98-3.91\left(\mathrm{~m}, 2 \mathrm{H},-\mathrm{OCH}_{2}\right), 2.70\left(\mathrm{~s}, 3 \mathrm{H},-\mathrm{CH}_{3}\right), 1.34(\mathrm{t}, \mathrm{J}$ : $\left.6.8 \mathrm{~Hz}, 3 \mathrm{H},-\mathrm{CH}_{3}\right) .{ }^{13} \mathrm{C}$ NMR $\left(125 \mathrm{MHz}, \mathrm{CDCl}_{3}\right) \delta(\mathrm{ppm})$ : 
178.3，175.7 (C=O), 163.1, 158.1, 144.7, 140.8, 137.2, $134.1,128.1,127.4,115.9,111.4,109.9,106.8,103.7\left(\mathrm{C}_{\mathrm{q}}\right.$ and $\left.\mathrm{C}_{\text {arom }}\right), 62.7\left(-\mathrm{OCH}_{2}\right), 24.0,13.8\left(-\mathrm{CH}_{3}\right)$. MS (ESI+) $m / z(\%): 343\left(100,[\mathrm{M}+\mathrm{H}]^{+}\right), 345\left(33,[\mathrm{M}+3 \mathrm{H}]^{+}\right)$. Anal. Calcd. for $\mathrm{C}_{18} \mathrm{H}_{15} \mathrm{ClN}_{2} \mathrm{O}_{3}$ (342.78).

6-(2-Ethoxyphenylamino)-7-chloro-2-methylquinoline-5,8-dione (3f) It was synthesized from 6,7-dichloro2-methylquinoline-5,8-dione (1) and 2-ethoxybenzenamine (2f) as purple powder by using the general procedure. Yield: $0.20 \mathrm{~g}, 56 \%, \mathrm{mp}: 205-206^{\circ} \mathrm{C}$. FTIR (ATR) $v\left(\mathrm{~cm}^{-1}\right)$ : 3334 $(-\mathrm{NH}), 3002,2947,2908\left(\mathrm{CH}_{\text {aliphatic }}\right), 1672$, $1607(\mathrm{C}=\mathrm{O}), 1580(\mathrm{C}=\mathrm{C}) .{ }^{1} \mathrm{H}$ NMR $\left(400 \mathrm{MHz}, C D C l{ }_{3}\right)$ $\delta$ (ppm): $8.33\left(\mathrm{~d}, \mathrm{~J}: 8.0 \mathrm{~Hz}, 1 \mathrm{H},-\mathrm{CH}_{\text {arom }}\right), 7.66(\mathrm{br} \mathrm{s}, 1 \mathrm{H}$, $-\mathrm{NH}), 7.51\left(\mathrm{~d}, \mathrm{~J}: 8.0 \mathrm{~Hz}, 1 \mathrm{H},-\mathrm{CH}_{\text {arom }}\right), 7.26-7.17(\mathrm{~m}, 1 \mathrm{H}$, $\left.-\mathrm{CH}_{\text {arom }}\right), 7.08-7.02\left(\mathrm{~m}, 1 \mathrm{H},-\mathrm{CH}_{\text {arom }}\right), 6.91-7.01(\mathrm{~m}$, $\left.2 \mathrm{H},-\mathrm{CH}_{\text {arom }}\right), 4.11\left(\mathrm{q}, \mathrm{J}: 14.0,7.0 \mathrm{~Hz}, 2 \mathrm{H},-\mathrm{OCH}_{2}\right), 2.83$ (s, $\left.3 \mathrm{H},-\mathrm{OCH}_{3}\right), 1.39$ (t, J: $\left.7.0 \mathrm{~Hz}, 3 \mathrm{H},-\mathrm{CH}_{3}\right) .{ }^{13} \mathrm{C}$ NMR $\left(100 \mathrm{MHz}, C D C l_{3}\right) \delta(\mathrm{ppm}): 180.0,176.1(\mathrm{C}=\mathrm{O}), 165.9$, 152.0, 148.1, 141.3, 134.9, 126.7, 126.3, 125.1, 124.9, 119.8, 115.6, $111.6\left(\mathrm{C}_{\mathrm{q}}\right.$ and $\left.\mathrm{C}_{\text {arom }}\right), 64.2\left(-\mathrm{OCH}_{2}-\right), 25.5,14.8(-$ $\mathrm{CH}_{3}$ ). MS (ESI-) $m / z(\%): 341\left(100,[\mathrm{M}-\mathrm{H}]^{-}\right), 343(22$, $\left.[\mathrm{M}+\mathrm{H}]^{-}\right)$. Anal. Calcd. for $\mathrm{C}_{18} \mathrm{H}_{15} \mathrm{ClN}_{2} \mathrm{O}_{3}(342.78)$.

6-(2,4-Dimethoxyphenylamino)-7-chloro-2-methylquinoline-5,8-dione (3g) It was synthesized from 6,7-dichloro2-methylquinoline-5,8-dione (1) and 2,4-dimethoxybenzenamine $(\mathbf{2 g})$ as brown powder by using the general procedure. Yield: $0.074 \mathrm{~g}, 20 \%$, mp: $186-187{ }^{\circ} \mathrm{C}$. FTIR $($ ATR $) v\left(\mathrm{~cm}^{-1}\right): 3224(-\mathrm{NH}), 3000,2996,2865\left(\mathrm{CH}_{\text {aliphatic }}\right)$, $1676,1652(\mathrm{C}=\mathrm{O}), 1607,1580(\mathrm{C}=\mathrm{C}) .{ }^{1} \mathrm{H} \mathrm{NMR}(400 \mathrm{MHz}$, $\left.C D \mathrm{Cl}_{3}\right) \delta(\mathrm{ppm}): 8.31\left(\mathrm{~d}, \mathrm{~J}: 7.8 \mathrm{~Hz}, 1 \mathrm{H},-\mathrm{CH}_{\text {arom }}\right), 7.49(\mathrm{~m}$, $2 \mathrm{H},-\mathrm{CH}_{\text {arom }}$ and $\left.-\mathrm{NH}\right), 7.02\left(\mathrm{~d}, \mathrm{~J}: 9.4 \mathrm{~Hz}, 1 \mathrm{H},-\mathrm{CH}_{\text {arom }}\right)$, 6.53-6.48 (m, $\left.2 \mathrm{H},-\mathrm{CH}_{\text {arom }}\right), 3.88\left(\mathrm{~s}, 3 \mathrm{H},-\mathrm{OCH}_{3}\right), 3.83$ $\left(\mathrm{s}, 3 \mathrm{H},-\mathrm{OCH}_{3}\right), 2.78\left(\mathrm{~s}, 3 \mathrm{H}, \mathrm{CH}_{3}\right) \cdot{ }^{13} \mathrm{C}$ NMR $(100 \mathrm{MHz}$, $\left.\mathrm{CDCl}_{3}\right) \delta(\mathrm{ppm}): 180.0,176.0(\mathrm{C}=\mathrm{O}), 165.9,159.3,154.4$, 148.2, 141.6, 134.9, 126.7, 126.6, 124.8, 119.4, 114.2, 103.4, $98.7\left(\mathrm{C}_{\mathrm{q}}\right.$ and $\left.\mathrm{C}_{\text {arom }}\right), 55.7\left(-\mathrm{OCH}_{3}\right), 25.5\left(-\mathrm{CH}_{3}\right)$. MS $(\mathrm{ESI}+) m / z(\%): 359\left(100,[\mathrm{M}+\mathrm{H}]^{+}\right), 361\left(34,[\mathrm{M}+3 \mathrm{H}]^{+}\right)$. Anal. Calcd. for $\mathrm{C}_{18} \mathrm{H}_{15} \mathrm{ClN}_{2} \mathrm{O}_{4}$ (358.78).

6-(2,5-Dimethoxyphenylamino)-7-chloro-2-methylquinoline-5,8-dione (3h) It was synthesized from 6,7-dichloro-2-methylquinoline-5,8-dione (1) and 2,5-dimethoxybenzenamine (2h) as purple powder by using the general procedure. Yield: $0.248 \mathrm{~g}, 67 \%$, mp: $176-$ $177^{\circ} \mathrm{C}$. FTIR (ATR) $v\left(\mathrm{~cm}^{-1}\right): 3226(-\mathrm{NH}), 2998,2967$, $2867\left(\mathrm{CH}_{\text {aliphatic }}\right), 1676,1652,(\mathrm{C}=\mathrm{O}), 1607,1578 .(\mathrm{C}=\mathrm{C})$. ${ }^{1} \mathrm{H}$ NMR $\left(500 \mathrm{MHz}, \mathrm{CDCl}_{3}\right) \delta(\mathrm{ppm}): 8.21$ (d, J: $7.8 \mathrm{~Hz}$, $\left.1 \mathrm{H},-\mathrm{CH}_{\text {arom }}\right), 7.49$ (br s, $\left.1 \mathrm{H},-\mathrm{NH}\right), 7.40(\mathrm{~d}, J: 7.8 \mathrm{~Hz}$, $\left.1 \mathrm{H},-\mathrm{CH}_{\text {arom }}\right), 6.75\left(\mathrm{~d}, \mathrm{~J}: 8.8 \mathrm{~Hz}, 1 \mathrm{H},-\mathrm{CH}_{\text {arom }}\right), 6.66(\mathrm{dd}, \mathrm{J}$ : 8.8, J: $\left.2.9 \mathrm{~Hz}, 1 \mathrm{H},-\mathrm{CH}_{\text {arom }}\right), 6.51$ (d, J: $\left.2.9 \mathrm{~Hz}, 1 \mathrm{H},-\mathrm{CH}_{3}\right)$, $3.88\left(\mathrm{~s}, 3 \mathrm{H},-\mathrm{OCH}_{3}\right), 3.73\left(\mathrm{~s}, 3 \mathrm{H},-\mathrm{OCH}_{3}\right), 2.71(\mathrm{~s}, 3 \mathrm{H}$,
$\left.\mathrm{CH}_{3}\right) .{ }^{13} \mathrm{C}$ NMR $\left(125 \mathrm{MHz}, \mathrm{CDCl}_{3}\right) \delta(\mathrm{ppm}): 179.8,176.0$ $(\mathrm{C}=\mathrm{O}), 165.9,152.9,148.3,146.9,141.1,134.9,134.8$, $126.7,126.6,124.8,115.8,111.4,111.3\left(\mathrm{C}_{\mathrm{q}}\right.$ and $\left.\mathrm{C}_{\text {arom }}\right)$, 55.8, $56.2\left(-\mathrm{OCH}_{3}\right), 25.5\left(-\mathrm{CH}_{3}\right)$. MS (ESI-) $\mathrm{m} / z(\%)$ : $357\left(100,[\mathrm{M}-\mathrm{H}]^{-}\right), 359\left(22,[\mathrm{M}+\mathrm{H}]^{-}\right)$. Anal. Calcd. for $\mathrm{C}_{18} \mathrm{H}_{15} \mathrm{ClN}_{2} \mathrm{O}_{4}(358.78)$.

6-(3,4-Dimethoxyphenylamino)-7-chloro-2-methylquinoline-5,8-dione (3i) It was synthesized from 6,7-dichloro2-methylquinoline-5,8-dione (1) and 3,4-dimethoxybenzenamine (2i) as dark red oil by using the general procedure. Yield: $0.274 \mathrm{~g}, 74 \%$. FTIR (ATR) $v\left(\mathrm{~cm}^{-1}\right): 3224$ (-NH), 3002, 2989, $2859\left(\mathrm{CH}_{\text {aliphatic }}\right), 1676,1652(\mathrm{C}=\mathrm{O})$, 1607, $1578(\mathrm{C}=\mathrm{C}) .{ }^{1} \mathrm{H}$ NMR $\left(500 \mathrm{MHz}, \mathrm{CDCl}_{3}\right) \delta(\mathrm{ppm})$ : $8.22\left(\mathrm{~d}, J: 7.8 \mathrm{~Hz}, 1 \mathrm{H},-\mathrm{CH}_{\text {arom }}\right), 7.57($ br s, $1 \mathrm{H},-\mathrm{NH}), 7.40$ $\left(\mathrm{d}, \mathrm{J}: 8.3 \mathrm{~Hz}, 1 \mathrm{H},-\mathrm{CH}_{\text {arom }}\right), 6.76\left(\mathrm{~d}, \mathrm{~J}: 8.8 \mathrm{~Hz}, 1 \mathrm{H},-\mathrm{CH}_{\text {arom }}\right)$, 6.63-6.59 (m, $\left.2 \mathrm{H},-\mathrm{CH}_{\text {arom }}\right), 3.84\left(\mathrm{~s}, 3 \mathrm{H},-\mathrm{OCH}_{3}\right), 3.80$ (s, 3H, $\left.-\mathrm{OCH}_{3}\right), 2.71\left(\mathrm{~s}, 3 \mathrm{H}, \mathrm{CH}_{3}\right) .{ }^{13} \mathrm{C}$ NMR $(125 \mathrm{MHz}$, $\left.\mathrm{CDCl}_{3}\right) \delta(\mathrm{ppm}): 180.1,176.1(\mathrm{C}=\mathrm{O}), 166.1,148.6,148.1$, 147.6, 141.1, 134.9, 130.2, 126.7, 124.6, 117.3, 114.6, 110.5, 109.2 $\left(\mathrm{C}_{\mathrm{q}}\right.$ and $\left.\mathrm{C}_{\text {arom }}\right), 56.1,56.0\left(-\mathrm{OCH}_{3}\right), 25.4\left(-\mathrm{CH}_{3}\right)$. MS (ESI-) $m / z(\%): 357\left(100,[\mathrm{M}-\mathrm{H}]^{-}\right), 359\left(38,[\mathrm{M}+\mathrm{H}]^{-}\right)$. Anal. Calcd. for $\mathrm{C}_{18} \mathrm{H}_{15} \mathrm{ClN}_{2} \mathrm{O}_{4}$ (358.78).

6-(3,5-Dimethoxyphenylamino)-7-chloro-2-methylquinoline-5,8-dione (3j) It was synthesized from 6,7-dichloro2-methylquinoline-5,8-dione (1) and 3,5-dimethoxybenzenamine (2) as dark red powder by using the general procedure. Yield: $0.190 \mathrm{~g}, 51 \%$, mp: $197-198{ }^{\circ} \mathrm{C}$. FTIR (ATR) $v\left(\mathrm{~cm}^{-1}\right): 3226(-\mathrm{NH}), 3002,2994,2853\left(\mathrm{CH}_{\text {aliphatic }}\right)$, 1678, $1652(\mathrm{C}=\mathrm{O}), 1607,1580(\mathrm{C}=\mathrm{C}) .{ }^{1} \mathrm{H}$ NMR $(500 \mathrm{MHz}$, $\left.\mathrm{CDCl}_{3}\right) \delta(\mathrm{ppm}): 8.22\left(\mathrm{~d}, \mathrm{~J}: 8.3 \mathrm{~Hz}, 1 \mathrm{H},-\mathrm{CH}_{\text {arom }}\right), 7.52(\mathrm{br}$ s, $1 \mathrm{H},-\mathrm{NH}), 7.40\left(\mathrm{~d}, J: 8.3 \mathrm{~Hz}, 1 \mathrm{H},-\mathrm{CH}_{\text {arom }}\right), 6.26(\mathrm{t}, \mathrm{J}$ : $\left.1.9 \mathrm{~Hz}, 1 \mathrm{H},-\mathrm{CH}_{\text {arom }}\right), 6.16\left(\mathrm{~d}, \mathrm{~J}: 2.4 \mathrm{~Hz}, 2 \mathrm{H},-\mathrm{CH}_{\text {arom }}\right), 3.71$ $\left(\mathrm{s}, 6 \mathrm{H},-\mathrm{OCH}_{3}\right), 2.71\left(\mathrm{~s}, 3 \mathrm{H}, \mathrm{CH}_{3}\right) .{ }^{13} \mathrm{C}$ NMR $(125 \mathrm{MHz}$, $\left.\mathrm{CDCl}_{3}\right) \delta(\mathrm{ppm}): 180.0,176.2(\mathrm{C}=\mathrm{O}), 166.1,160.5,147.9$, 140.9, 138.8, 134.9, 126.9, 124.7, 116.3, 103.00, 102.9, 98.2, $98.1\left(\mathrm{C}_{\mathrm{q}}\right.$ and $\left.\mathrm{C}_{\text {arom }}\right), 55.4,55.6\left(-\mathrm{OCH}_{3}\right), 25.5\left(-\mathrm{CH}_{3}\right)$. MS $(\mathrm{ESI}+) \mathrm{m} / z(\%): 359\left(100,[\mathrm{M}+\mathrm{H}]^{+}\right)$. Anal. Calcd. for $\mathrm{C}_{18} \mathrm{H}_{15} \mathrm{ClN}_{2} \mathrm{O}_{4}$ (358.78).

\section{In vitro antimicrobial activity}

\section{Determination of minimum inhibitory concentrations (MIC)}

Antimicrobial activity against Staphylococcus aureus ATCC 29213, Staphylococcus epidermidis ATCC 12228, Escherichia coli ATCC 25922, Klebsiella pneumoniae ATCC 4352, Pseudomonas aeruginosa ATCC 27853, Proteus mirabilis ATCC 14153, Enterococcus faecalis ATCC 29212, Candida albicans ATCC 10231, Candida parapsilosis ATCC 22019, and Candida tropicalis ATCC 750 was determined by the microbroth dilutions technique using the Clinical Laboratory Standards Institute (CLSI) recommendations [23, 24]. Mueller-Hinton broth for 
bacteria and RPMI-1640 medium for yeast strain were used as the test medium. Serial twofold dilutions ranging from 5000 to $2.44 \mu \mathrm{g} / \mathrm{mL}$ were prepared in medium. The inoculum was prepared using a $4-6 \mathrm{~h}$ broth culture of each bacteria type and $24 \mathrm{~h}$ culture of yeast strains adjusted to a turbidity equivalent to $0.5 \mathrm{McFarland}$ standard, diluted in broth media to give a final concentration of $5 \times 10^{5} \mathrm{cfu} / \mathrm{mL}$ for bacteria and $5 \times 10^{3} \mathrm{cfu} / \mathrm{mL}$ for yeast in the test tray. The trays were covered and placed into plastic bags to prevent evaporation. The trays containing Mueller-Hinton broth were incubated at $35{ }^{\circ} \mathrm{C}$ for $18-20 \mathrm{~h}$ while the trays containing RPMI-1640 medium were incubated at $35{ }^{\circ} \mathrm{C}$ for $46-50 \mathrm{~h}$. The MIC was defined as the lowest concentration of compound giving complete inhibition of visible growth. As control, antimicrobial effects of the solvents were investigated against test microorganisms. According to values of the controls, the results were evaluated. The MIC values of the compounds are given in Table 1.

\section{Conclusions}

In summary, in order to obtain antimicrobial lead compounds, (alkoxy)phenylamino-chloro-2-methylquinoline-5,8-dione derivatives $\left(\mathbf{3 a}-\mathbf{j}, \mathbf{3} \mathbf{a}^{\prime}, \mathbf{3} \mathbf{e}^{\prime}\right)$ were synthesized. Three active compounds (3a, $\mathbf{3} \mathbf{b}$, and $\mathbf{3 h}$ ) exhibited better activity than that of the reference antimicrobial compound. In vitro antimicrobial study of the newly synthesized compounds was evaluated in a panel of three fungi and seven bacterial strains (three Grampositive and four Gram-negative bacteria). As a result, the compounds ( $\mathbf{3 a}, \mathbf{3 b}$, and $\mathbf{3 h}$ ) were identified as the hits with the strong antibacterial efficiency against the human originated pathogens S. epidermidis and E. faecalis with some minimal inhibitory concentration values. These results suggest that these substances have potential for exploring the design of new antimicrobial prototypes against Gram-positive bacteria. Moreover, the results might encourage the synthesis of new 6-substituted-5,8-quinolinedione analogs for improving potential. Comparing the activity of the methoxy and ethoxy derivatives of QD compounds, one can notice that the introduction of the amines with methoxy group leads to positive changes in the antibacterial activity against tested pathogens.

\section{Authors' contributions}

The author read and approved the final manuscript.

\section{Acknowledgements}

I also gratefully thank to Dr. Emel Marataci Kara and Prof. Dr. Berna Özbek Çelik for providing me the biological data of synthesized compounds from the Department of Pharmaceutical Microbiology Department in the Pharmacy Faculty at Istanbul University.
Competing interests

The author declares that there is no competing interests.

Availability of data and materials

All data are fully available without restriction.

Consent for publication

The author declares that the copyright belongs to the journal.

Ethics approval and consent to participate

Not applicable.

\section{Funding}

No funding was received.

\section{Publisher's Note}

Springer Nature remains neutral with regard to jurisdictional claims in published maps and institutional affiliations.

Received: 11 January 2018 Accepted: 12 February 2018

Published online: 23 February 2018

References

1. Yang H, Wang HW, Zhu TW, Yu LM, Chen JW, Wang LX et al (2017) Syntheses and antibacterial activity of soluble 9-bromo substituted indolizinoquinoline-5,12-dione derivatives. Eur J Med Chem 127:166-173

2. Seradj H, Cai W, Erasga NO, Chenault DV, Knuckles KA, Ragains JR et al (2004) Total synthesis of novel 6-substituted lavendamycin antitumor agents. Org Lett 6(4):473-476

3. Novais JS, Campos VR, Silva ACJA, de Souza MCBV, Ferreira VF, Keller VGL et al (2017) Synthesis and antimicrobial evaluation of promising 7-arylamino-5,8-dioxo-5,8-dihydroisoquinoline-4-carboxylates and their halogenated amino compounds for treating Gram-negative bacterial infections. RSC Adv 7(30):18311-18320

4. Ryu CK, Kim DH, Kim DH, Lee IK, Kim SH (1996) 6-[(N-2,3-dichlorophenyl) amino]-7-chloro-5,8-quinolinedione treatment of candidiasis in normal mice. Arch Pharm Res 19(3):197-200

5. Ryu CK, Kang HY, Yi YJ, Lee CO (2000) Cytotoxic activities of 6-aylamino7-halo-5,8-quinolinediones against human tumor cell lines. Arch Pharm Res 23(1):42-45

6. Behforouz M, Cai W, Stocksdale MG, Lucas JS, Jung JY, Briere D et al (2003) Novel lavendamycin analogues as potent HIV-reverse transcriptase inhibitors: synthesis and evaluation of anti-reverse transcriptase activity of amide and ester analogues of lavendamycin. J Med Chem 46(26):5773-5780

7. Bayrak N, Yildirim H, Tuyun AF, Kara EM, Celik BO, Gupta GK et al (2017) Synthesis, computational study, and evaluation of in vitro antimicrobial, antibiofilm, and anticancer activities of new sulfanyl aminonaphthoquinone derivatives. Lett Drug Des Discov 14(6):647-661

8. Bayrak N, Yildirim H, Tuyun AF, Kara EM, Celik BO, Gupta GK (2016) Synthesis, biological, and computational study of naphthoquinone derivatives containing heteroatoms. J Chem Soc Pak 38(6):1211-1221

9. Scott DE, Coyne AG, Hudson SA, Abel C (2012) Fragment-based approaches in drug discovery and chemical biology. Biochemistry 51:4990-5003

10. Bolzan AD, Bianchi MS (2001) Genotoxicity of streptonigrin: a review. Mutat Res Rev Mutat 488(1):25-37

11. Hassani M, Cai W, Holley DC, Lineswala JP, Maharjan BR, Ebrahimian GR et al (2005) Novel lavendamycin analogues as antitumor agents: synthesis, in vitro cytotoxicity, structure-metabolism, and computational molecular modeling studies with $\mathrm{NAD}(\mathrm{P}) \mathrm{H}$ : quinone oxidoreductase 1. J Med Chem 48(24):7733-7749

12. Boger DL, Yasuda M, Mitscher LA, Drake SD, Kitos PA, Thompson SC (1987) Streptonigrin and lavendamycin partial structures-probes for the minimum, potent pharmacophore of streptonigrin, lavendamycin, and synthetic quinoline-5,8-diones. J Med Chem 30(10):1918-1928 
13. Musiol R, Magdziarz T, Kurczyk A (2011) Quinoline scaffold as a privileged substructure in antimicrobial drugs, vol 3. Formatex, Microbiology book series. Badajoz, pp 72-83

14. Lederberg J (2000) Infectious history. Science 288(5464):287-293

15. Singh SR, Krishnamurthy NB, Mathew BB (2014) A review on recent diseases caused by microbes. J Appl Environ Microbiol 2(4):106-115

16. WHO (2017) Methods and data sources for country—level causes of death 2000-2015. WHO, Geneva

17. WHO (2017) WHO publishes list of bacteria for which new antibiotics are urgently needed. WHO, Geneva

18. Yıldırım H, Bayrak N, Tuyun AF, Kara EM, Celik BÖ, Gupta GK (2017) 2,3-Disubstituted-1,4-naphthoquinones containing an arylamine with trifluoromethyl group: synthesis, biological evaluation, and computational study. RSC Adv 7:25753-25764

19. Lee HJ, Kim JS, Park SY, Suh ME, Kim HJ, Seo EK et al (2004) Synthesis and cytotoxicity evaluation of 6,11-dihydro-pyridazoand 6,11-dihydro-pyrido[2,3-blphenazine-6, 11-diones. Bioorgan Med Chem 12(7):1623-1628
20. Shaikh IA, Johnson F, Grollman AP (1986) Streptonigrin. 1. Structureactivity-relationships among simple bicyclic analogs-rate dependence of DNA-degradation on quinone reduction potential. J Med Chem 29(8):1329-1340

21. Yanni AS, Khalil ZH (1991) Synthesis and application of some new furoquinolinediones as bactericides. Collect Czech Chem C 56(3):706-711

22. Ryu CK, Kim HJ (1994) The synthesis of 6-(N-arylamino)-7-chloro-5,8-quinolinedione derivatives for evaluation of antifungal activities. Arch Pharm Res 17(3):139-144

23. Clinical and Laboratory Standards Institute (1997) Reference method for broth dilution antifungal susceptibility testing of yeasts. Approved standard, M27-A2, 2nd edn. Wayne, CLSI

24. Clinical and Laboratory Standards Institute (2006) Methods for dilution antimicrobial susceptibility tests for bacteria that grow aerobically. Approved standard, M7-A7, 7th edn. Wayne, CLS

\section{Submit your manuscript to a SpringerOpen ${ }^{\circ}$ journal and benefit from:}

- Convenient online submission

- Rigorous peer review

- Open access: articles freely available online

- High visibility within the field

- Retaining the copyright to your article

Submit your next manuscript at $\boldsymbol{\nabla}$ springeropen.com 